\title{
Isoniazid- and Streptomycin-Resistant Miliary Tuberculosis Complicated by Intracranial Tuberculoma in a Japanese Infant
}

\author{
Naruhiko Ishiwada, ${ }^{1}$ Osamu Tokunaga, ${ }^{2}$ Koo Nagasawa, ${ }^{3}$ Keiko Ichimoto, ${ }^{3}$ \\ Kaori Kinoshita, ${ }^{3}$ Haruka Hishiki ${ }^{3}$ and Yoichi Kohno ${ }^{3}$ \\ ${ }^{1}$ Division of Control and Treatment of Infectious Diseases, Chiba University Hospital, Chiba City, Chiba, Japan \\ ${ }^{2}$ Department of Pediatrics, National Hospital Organization Minami-Kyoto Hospital, Joyo City, Kyoto, Japan \\ ${ }^{3}$ Department of Pediatrics, Chiba University Graduate School of Medicine, Chiba City, Chiba, Japan
}

In Japan, the incidence of severe pediatric tuberculosis (TB) has decreased dramatically in recent years. However, children in Japan can still have considerable opportunities to contract TB infection from adult TB patients living nearby, and infants infected with TB may develop severe disseminated disease. A 3-month-old girl was admitted to our hospital with dyspnea and poor feeding. After admission, miliary TB and multiple brain tuberculomas were diagnosed. Anti-tuberculous therapy was initiated with streptomycin $(\mathrm{SM})$, isoniazid $(\mathrm{INH})$, rifampicin and pyrazinamide. Symptoms persisted after starting the initial treatment and mycobacterial cultures of gastric fluid remained positive. Drug sensitivity testing revealed the TB strain isolated on admission as completely resistant to INH and SM. Treatments with INH and SM were therefore stopped, and treatment with ethambutol and ethionamide was started in addition to rifampicin and pyrazinamide. After this change to the treatment regimen, symptoms and laboratory data gradually improved. The patient was treated with these four drugs for 18 months, and then pyrazinamide was stopped. After another 2 months, ethambutol was stopped. Treatment of tuberculosis was completed in 24 months. No adverse effects of these anti-TB drugs were observed. The patient achieved a full recovery without any sequelae. On the other hand, the infectious source for this patient remained unidentified, despite the extensive contact investigations. The incidence of drug-resistant TB is increasing in many areas of the world. Continuous monitoring for pediatric patients with drug-resistant TB is therefore needed.

Keywords: infant; isoniazid-resistant; Japan; miliary tuberculosis; tuberculoma

Tohoku J. Exp. Med., 2013 Mar, 229 (3), 221-225. C 2013 Tohoku University Medical Press

\section{Introduction}

Recently, severe cases of pediatric tuberculosis (TB) have decreased dramatically in Japan, thanks to the high coverage rate for infantile BCG vaccination and environmental changes (Tuberculosis Surveillance Center 2012). However, TB remains one of the most common infectious diseases among the elderly in Japan, and the prevalence of TB disease remains relatively high, with Japan classified as an "intermediate-prevalence" country for TB. Despite joint efforts by the private sector and public health departments to control $\mathrm{TB}$, the treatment success rate for TB disease remains at $78.6 \%$ in Japan (Tuberculosis Research Committee 2007). One reason for treatment failure is initial drug-resistance to anti-TB drugs among newly diagnosed patients with TB. Accumulation of clinical information about drug-resistant TB is thus very informative. We report herein a pediatric patient with miliary TB complicated by intracranial tuberculoma caused by a drug-resistant strain.

\section{Clinical Report}

A 3-month-old girl was referred to our hospital with a 3-week history of cough, dyspnea, and poor feeding on September 9, 2009. She had initially been treated with expectorants and erythromycin for presumed upper respiratory tract infection, but no improvement was attained. The patient was the first-born child, delivered vaginally at full term after a complication-free pregnancy with a birth weight of $3.5 \mathrm{~kg}$. No significant medical history or regular medication use prior to this illness was elicited from the parents, and no vaccinations had been administered, including BCG. No family history of pulmonary or extrapulmonary tuberculosis was noted. Her father was Japanese and her mother was Chinese, and several of the mother's relatives had visited with the patient during the neonatal period.

On examination, body weight was $5.7 \mathrm{~kg}$, within the normal range for her age. Nutritional status was normal.

Received December 26, 2012; accepted February 7, 2013. Published online March 6, 2013; doi: 10.1620/tjem.229.221.

Correspondence: Naruhiko Ishiwada, Division of Control and Treatment of Infectious Diseases, Chiba University Hospital, 1-8-1 Inohana,

Chuo-ku, Chiba, Chiba 260-8677, Japan.

e-mail: ishiwada@ faculty.chiba-u.jp 


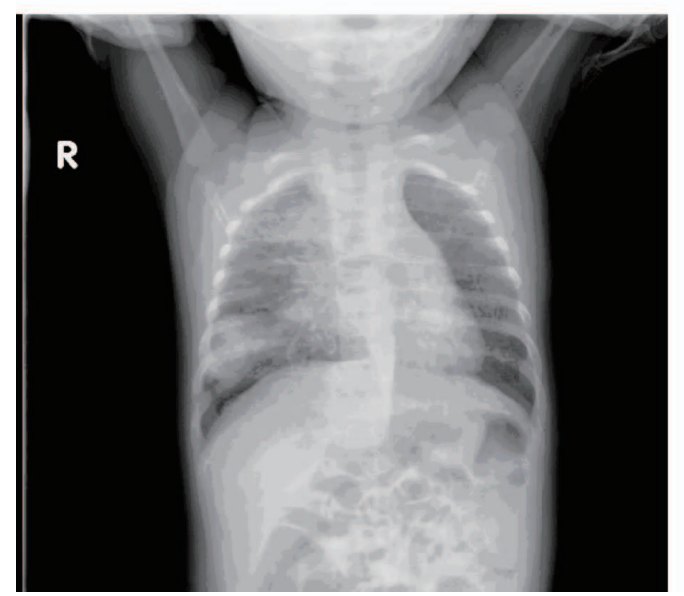

b

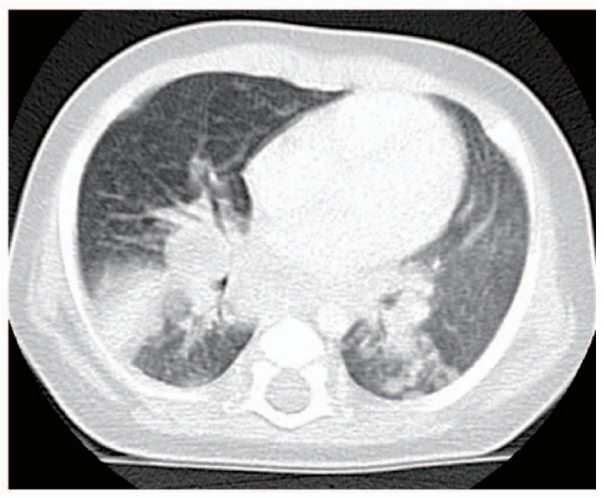

Fig. 1. Chest radiography and high-resolution computed tomography (CT) of the thorax on admission.

a) Chest radiography reveals round-shaped shadows in the lower lung fields with diffuse, bilateral, small nodules.

b) Similarly, widespread, micronodular, hyperdense formations are seen throughout the lungs on high-resolution CT.

She had a temperature of $37^{\circ} \mathrm{C}$, heart rate was regular $(130$ beats $/ \mathrm{min})$, and blood pressure was normal. Transcutaneous oxygen saturation was $96 \%$ on room air, and respiratory rate was 40 breaths/min. Her consciousness was clear. Breath sounds were weak in the left lower chest, and crackles and wheezes were heard on both sides. Results of all other clinical examination were unremarkable. Results of laboratory tests on admission showed: white blood cell count, 18,400 / $\mu \mathrm{l}$; hemoglobin, $12.1 \mathrm{~g} / \mathrm{dl}$; hematocrit, $35.2 \%$; platelet count, $34.9 \times 10^{4} / \mu \mathrm{l}$; and C-reactive protein (CRP), $3.1 \mathrm{mg} / \mathrm{dl}$. HIV-1 serology was non-reactive. Chest radiography revealed a round-shaped shadow in the lower lung field with diffuse, bilateral, small nodules (Fig. 1a) and high-resolution computed tomography of the thorax (Fig. 1b) suggested pulmonary TB with hematogenous dissemination. Ziehl-Neelsen stain-positive organisms were detected in early morning gastric aspirate (Gaffky scale 4) on hospital day 2. Polymerase chain reaction (PCR) testing for Mycobacterium tuberculosis complex and mycobacterial culture on gastric aspirates yielded positive results. The tuberculin skin test and QuantiFERON-TB test also showed positive results (ESAT-6, $2.365 \mathrm{IU} / \mathrm{ml}$; CFP-10, $9.534 \mathrm{IU} /$ $\mathrm{ml})$.

Brain magnetic resonance imaging (MRI) suggested multiple brain tuberculomas (Fig. 2). Lumbar puncture was performed to evaluate the possibility of meningitis. Cerebrospinal fluid (CSF) appeared normal and adenosine deaminase levels in CSF were not elevated. Smears, cultures and PCR testing of a CSF sample showed negative results for TB. Electroencephalography showed no abnormal waves. Family members were investigated for TB disease, but neither the parents nor the paternal grandparents showed any abnormalities on chest radiography film and the source of TB infection was not identified. The patient was diagnosed with miliary TB with multiple brain tuberculomas.
Anti-tuberculous therapy with streptomycin (SM) (30 $\mathrm{mg} / \mathrm{kg}$ intramuscularly, once daily), INH $(10 \mathrm{mg} / \mathrm{kg}$ orally, once daily), rifampicin (RFP) (15 mg/kg orally, once daily) and pyrazinamide (PZA) $(15 \mathrm{mg} / \mathrm{kg}$ orally, once daily) were started immediately. However, symptoms persisted with intermittent low-grade fever. CRP levels remained positive. Acid-fast stain testing and mycobacterial cultures for gastric fluid continued to show positive results. Drug sensitivity testing revealed that the TB strain isolated on admission was completely resistant to INH and SM (Table 1). In follow-up brain MRI performed the same day, brain tuberculomas showed a significant expansion in size. Treatment with INH and SM was therefore stopped and a treatment regimen comprising ethambutol (EB) $(25 \mathrm{mg} / \mathrm{kg}$ orally, once daily) and ethionamide (TH) (20 mg/kg orally, twice daily) in addition to RFP and PZA was started from October 19. After changing the treatment regimen, clinical symptoms, laboratory data and radiological findings gradually normalized. Acid bacilli stain tests yielded negative results for 3 consecutive days from December 12 to December 14 and the patient was discharged from hospital. She was treated with the four drugs for 18 months in total before stopping PZA. After another 2 months, EB was stopped. Treatment of TB was completed within 24 months. No adverse effects for these anti-TB drugs were observed. The patient did not experience any epileptic attacks and achieved full recovery without any neurological sequelae.

\section{Discussion}

In this study, we have reported a pediatric patient that was diagnosed as miliary TB with multiple brain tuberculomas. Brain tuberculoma is a rare form of extra-pulmonary TB that is occasionally seen in patients with miliary TB and no meningitis. Miliary TB and related complications in pediatric patients have not frequently been seen in Japan (Tuberculosis Surveillance Center 2012). Radiological 

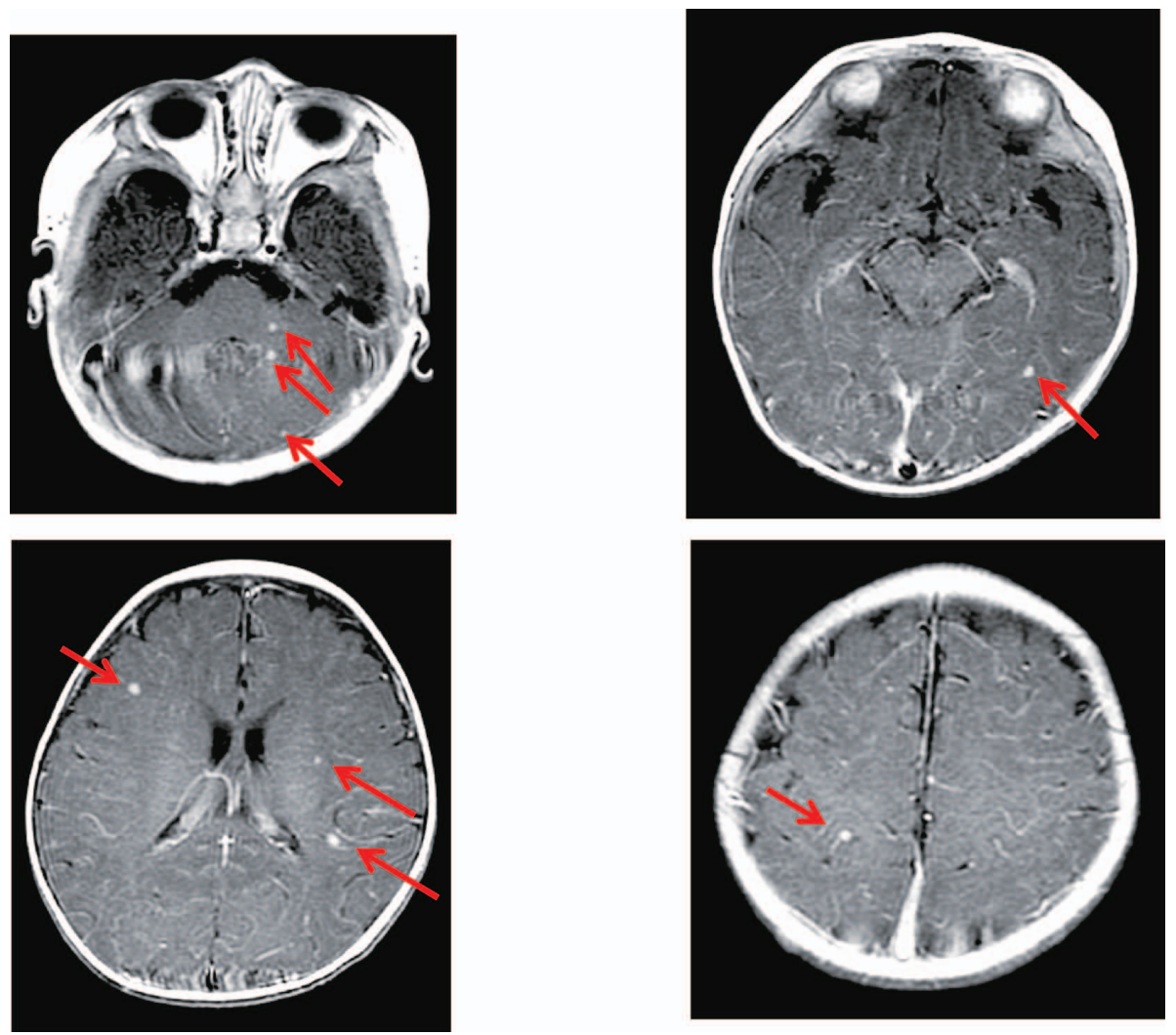

Fig. 2. Brain magnetic resonance imaging (MRI).

Multiple small rounded lesions with ring enhancement were observed in gadolinium-enhanced T1-weighted magnetic resonance imaging of the brain. $(\rightarrow)$

Table 1. Sensitivity to anti-tuberculous drugs.

\begin{tabular}{llrl}
\hline Streptomycin & $(\mathrm{SM})$ & $\geq 128 \mu \mathrm{g} / \mathrm{ml}$ & $\underline{\text { Resistant }}$ \\
Kanamycin & $(\mathrm{KM})$ & $2 \mu \mathrm{g} / \mathrm{ml}$ & Sensitive \\
Levofloxacin & $(\mathrm{LVFX})$ & $0.125 \mu \mathrm{g} / \mathrm{ml}$ & Sensitive \\
Rifampicin & $(\mathrm{RFP})$ & $\leq 0.03 \mu \mathrm{g} / \mathrm{ml}$ & Sensitive \\
Cycloserine & $(\mathrm{CS})$ & $\leq 30 \mu \mathrm{g} / \mathrm{ml}$ & Sensitive \\
Enviomycin & $(\mathrm{EVM})$ & $\leq 20 \mu \mathrm{g} / \mathrm{ml}$ & Sensitive \\
$\underline{\text { Isoniazid }}$ & $(\mathrm{INH})$ & $\underline{\mu \mathrm{g} / \mathrm{ml}}$ & $\underline{\text { Resistant }}$ \\
Paraaminosalicylic acid & $(\mathrm{PAS})$ & $\leq 0.5 \mu \mathrm{g} / \mathrm{ml}$ & Sensitive \\
Ethambutol & $(\mathrm{EB})$ & $2 \mu \mathrm{g} / \mathrm{ml}$ & Sensitive \\
Pyrazinamide & $(\mathrm{PZA})$ & $\leq 1,000 \mu \mathrm{g} / \mathrm{ml}$ & Sensitive \\
Ethionamide & $(\mathrm{TH})$ & $\leq 20 \mu \mathrm{g} / \mathrm{ml}$ & Sensitive \\
\hline
\end{tabular}

findings for brain tuberculomas are nonspecific. Multiple small, round lesions with annular enhancement were observed on brain MRI in the present patient. Clinical manifestations of brain tuberculomas depend largely on their location. In general, symptoms are initially silent and gradually develop within weeks to months (Rock et al. 2008). The most important factors affecting the prognosis for central nervous system (CNS) tuberculosis are early diagnosis and prompt initiation of anti-TB therapy (Tinsa et al. 2010). The TB strain isolated from this patient was identified as resistant to INH and SM. The incidence of drug-resistant TB is increasing in many areas of the world. The TB Research Committee (Ryoken), a nationwide coalition of TB hospitals in Japan, has conducted regular nationwide drug-resistance surveys. The Committee reported that the rate of completely INH-resistant strains among newly diagnosed cases of all ages and in patients $<19$ years old were $5.0 \%$ and $12.1 \%$, respectively, in 2009 (Tuberculosis Surveillance Center 2011). Drug-resistant TB is more prevalent among pediatric patients than among adult patients in Japan. Ito (1999) and Mori and Yokota (2004) reported prevalences for INH resistance of $4.4 \%$ and $7 \%$, respec- 
tively, among culture-confirmed cases of pediatric TB in a single hospital-based study. According to the registry information system for tuberculosis in Japan, the prevalences of drug-resistant strains to any TB drugs and to INH among all pediatric TB cases from 2008 to 2009 were $17 \%$ and $6 \%$, respectively, based on drug sensitivity test results for pediatric patients and their index cases (Tokunaga and Kato 2011). Insufficient evidence has been accumulated regarding the treatment of drug-resistant TB in children, so the most effective treatment regimens remain unclear. Several guidelines for drug-resistant TB in children have been published recently (Schaaf and Marais 2011; Al-Dabbagh et al. 2011). According to these guidelines, with extensive pulmonary or disseminated extra-pulmonary disease, a minimum of 4 active drugs should be included in the treatment regimen. The Japanese Society for TB recently published guidelines for the treatment of TB. Intensive pharmacotherapy, comprising INH, RFP, PZA and SM (or EB), is recommended if patients are $<80$ years old and have normal liver function (Treatment Committee of the Japanese Society for Tuberculosis 2011). INH, RFP and PZA are familiar to both chest physicians and pediatricians. These three drugs are key drugs for current anti-TB regimens and are used in virtually all patients unless drug resistance is known to be present. SM is also frequently used for pediatric miliary or meningeal $\mathrm{TB}$ patients, although this drug should be given intramuscularly. The present patient was initially treated using INH, RFP, PZA and SM. After drug resistance to INH and SM were revealed, we switched these 2 drugs to EB and TH. For many years, EB was not recommended for use in children for fear of ocular toxicity, specifically optic neuritis and color blindness. However, recent clinical studies have shown that the incidence of eye toxicity with EB is extremely low in children, if it occurs at all (Thee et al. 2007). Clinicians thus should not hesitate to use EB as indicated, particularly if there is an increased likelihood of INH-resistant TB (Starke 2001). Generally, penetration of INH and PZA into spinal fluid is good, while penetration of RFP, EB, and SM is poorer. However, all of these drugs can penetrate into spinal fluid in the presence of active inflammation.

In South Africa, TH is frequently used as a fourth drug in anti-TB treatment regimens, particularly when there is a concern about tuberculous meningitis. TH can pass the blood-brain barrier very well (Donald 2010). On the other hand, TH is one of the second-line drugs against drug-resistant TB (Schaaf and Marais 2011; Al-Dabbagh et al. 2011) and can cause liver toxicity. As TH has a chemical structure similar to that of INH, TH resistance should be suspected when INH resistant is present. Fluoroquinolones are frequently used in the treatment of adult TB disease, particularly when drug resistance is present. Most experts on pediatric TB agree with the use of these agents to treat childhood multidrug-resistant TB cases when other bactericidal drugs are not readily available. In terms of the duration of therapy, World Health Organization guidelines rec- ommend continuing therapy against multi-drug resistant TB for a minimum of 18 months after the first negative culture (World Health Organization 2009). We extended the duration of therapy to 24 months, as our patient showed disseminated TB lesions including CNS tuberculomas. The treatment period of anti-TB drugs, especially PZA in our patient was longer than the standard treatment period of each antiTB drugs. We regularly monitored the patient for toxic reactions to medications and fortunately, no adverse effects for anti-TB drugs were observed. In terms of the appropriate treatment period of pediatric patients with disseminated TB including CNS lesions caused by multidrug-resistant $\mathrm{TB}$, further discussion will be needed.

The infectious source for this patient remained unidentified, although the public health department undertook precise contact investigations. However, because the variable numbers of tandem repeats (VNTR) pattern in this patient was not a common one in Japan (data not shown), this baby might have developed TB infection following contact with her mother's relatives from China.

In conclusion, we encountered an infant with miliary TB and multiple brain tuberculomas caused by a SM- and INH-resistant strain. The combination therapy including RFP, PZA, EB and TH was effective for this patient and she was cured without any sequelae. In Japan, the frequency of severe pediatric TB has been decreasing dramatically in recent years. However, children in Japan can still have considerable opportunities to contract TB infection from adult TB patients living nearby, and infants infected with TB may develop severe disseminated disease, as in this case. Continuous monitoring for pediatric and drug-resistant TB cases is needed.

\section{Conflict of Interest}

All authors report no conflict of interest.

\section{References}

Al-Dabbagh, M., Lapphra, K., Mcgloin, R., Inrig, K., Schaaf, H.S., Marais, B.J., Sauve, L., Kitai, I. \& Kollmann, T.R. (2011) Drug-resistant tuberculosis: pediatric guidelines. Pediatr. Infect. Dis. J., 30, 501-505.

Donald, P.R. (2010) Cerebrospinal fluid concentration of antituberculosis agents in adults and children. Tuberculosis, 90, 279-292.

Ito, M. (1999) Drug resistant tuberculosis and pediatric tuberculosis. Shouninaika, 31, 69-73.

Mori, M. \& Yokota, S. (2004) Strategy for drug-resistant tuberculosis in childhood. Shounika, 45, 519-522.

Rock, R.B., Olin, M., Baker, C.A., Molitor, T.W. \& Peterson, P.K. (2008) Central nervous system tuberculosis: pathogenesis and clinical aspects. Clin. Microbiol. Rev., 21, 243-261.

Schaaf, H.S. \& Marais, B.J. (2011) Management of multidrugresistant tuberculosis in children: a survival guide for paediatricians. Paediatr. Respir. Rev., 12, 31-38.

Starke, J.R. (2001) Childhood tuberculosis: treatment strategies and recent advances. Pediatric. Respir. Rev., 2, 103-112.

Thee, S., Detjen, A., Quarcoo, D., Wahn, U. \& Magdorf, K. (2007) Ethambutol in paediatric tuberculosis: aspects of ethambutol serum concentration, efficacy and toxicity in children. Int. J. Tuberc. Lung Dis., 11, 965-971. 
The Treatment Committee of the Japanese Society for Tuberculosis. (2011) Review of "Standard for Tuberculosis Care"2008. Kekkaku, 86, 29-36.

Tinsa, F., Essaddam, L., Fitouri, Z., Boussetta, K., Becher, S.B. \& Bousnina, S. (2010) Central system nervous tuberculosis in infants. J. Child Neurol., 25, 102-106.

Tokunaga, O. \& Kato, S. (2011) Research on the registered childhood tuberculosis disease cases in Japan 2008 2009. Report of the research on emerging and re-emerging infectious diseases (H21-Shinko-Ippan-016) by grants from the Ministry of Health, Labor and Welfare of Japan 2010, 83-92.

Tuberculosis Research Committee (Ryoken) (2007) Drug-resistant
Mycobacterium tuberculosis in Japan: a nationwide survey, 2002. Int. J. Tuberc. Lung Dis., 11, 1129-1135.

Tuberculosis Surveillance Center (2011) Tuberculosis annual report 2009. Series 7 condition of TB (2). Kekkaku, 86, 883-887.

Tuberculosis Surveillance Center (2012) Tuberculosis annual report 2010. Series 3 childhood tuberculosis. Kekkaku, 87, 549-553.

World Health Organization (2009) Treatment of tuberculosis guidelines fourth edition. Available at: http://whqlibdoc.who. int/publications/2010/9789241547833_eng.pdf. [Cited December 2012]. 